CHAPTER XV

CLUSTERING OF QUASI-STELLAR OBJECTS 


\title{
CLUSTERING OF QUASARS FROM THE ROE/ESO LARGE-SCALE AQD SURVEY FOR QUASARS
}

\author{
Roger G. Clowes ${ }^{1}$, Angela Iovino ${ }^{2}$ and Peter Shaver ${ }^{2}$ \\ ${ }^{1}$ Royal Observatory, Edinburgh, Scotland \\ 2European Southern Observatory, Garching bei München, FRG
}

\begin{abstract}
$\underline{\text { Abstract }}$
The new ROE/ESO large-scale AQD survey for quasars forms a connected area of $\sim 200 \mathrm{deg}^{2}$ near the south galactic pole, and has resulted in the discovery of a total number of quasar candidates that is comparable to the number previously published from all other sources (see the poster paper by Iovino, Clowes \& Shaver at this conference). In this paper we describe the first results of a three-dimensional self-clustering analysis of $\sim 1100$ "high-probability" candidates occupying the assigned-redshift band of 1.8 to 2.4. Although the analysis is sensitive to very weak clustering we find no evidence that quasars are distributed in any way other than randomly. The implications of this result are discussed.
\end{abstract}

\section{Introduction}

One of the main difficulties of using quasars as cosmological indicators has, for many years, been the lack of large, complete samples. However, in the ROE/ESO survey for quasars, we have now successfully completed a very important stage in the assembly of such samples.

Briefly, seven low-dispersion, IIIa-J objective-prism plates from the UK Schmidt Telescope (UKST) have been measured with the COSMOS measuring machine (MacGillivray \& Stobie 1984) at ROE, and the data processed with the software for Automated Quasar Detection (AQD - Clowes, Cooke \& Beard 1984, Clowes 1986) to find the quasars. The seven plates form a connected area of $\sim 200$ $\operatorname{deg}^{2}$ near the south galactic pole, and the total number of newly-discovered quasar candidates is comparable to the number published from all other sources since the first quasars were discovered. Full details of the progress and aims of the ROE/ESO survey are given in the poster paper by Iovino, Clowes \& Shaver at this conference.

With such a large number of new candidates the ROE/ESO survey ideally needs a long programme of follow-up spectroscopy both for confirmation and for obtaining accurate redshifts. In this paper on the clustering properties, however, we circumvent the present absence of such spectroscopy by restricting our 
samples to the "high-probability" candidates from the five best plates.

The ability to grade the candidates in order of probability of being quasars is a very useful feature (it has something in common with the expert-system approach used in machine intelligence). It means that we can produce high-probability subsets with surface densities of $\sim 10 \mathrm{deg}^{-2}-$ more than would be found in a typical visual search -, with minimal need for access to large telescopes. There are, of course, many further quasars in the lower-probability grades.

\section{The clustering analysis - 3-D power spectrum analysis}

We use the method of power spectrum analysis (PSA) to test for clustering. The method was developed by Webster (1976) with the particular aim of distinguishing a population that is weakly clustered from one that is randomly distributed. A brief summary of the theory of PSA is given by Clowes (1986).

To obtain the third coordinate for 3-D PSA the cosmological hypothesis of redshifts is assumed and a particular cosmological model is adopted $-q_{0}=0$, $\mathrm{H}_{\mathrm{O}}=100 \mathrm{~h} \mathrm{kms}^{-1} \mathrm{Mpc}^{-1}$.

First we produced the high-probability subsets in the following five fields.

\begin{tabular}{|c|c|c|c|c|c|c|}
\hline $\begin{array}{l}\text { ESO/SERC } \\
\text { field }\end{array}$ & $\begin{array}{l}\text { UKST } \\
\text { plate }\end{array}$ & \multicolumn{2}{|c|}{ Plate centre $(1950)$} & N1 & N2 & N3 \\
\hline 295 & UJ6536P & 005200 & -400000 & 399 & 328 & 284 \\
\hline 296 & UJ5406P & $01 \quad 1800$ & -400000 & 219 & 213 & 171 \\
\hline 297 & UJ4514P & 014400 & -400000 & 407 & 380 & 308 \\
\hline 351 & UJ6528P & 004800 & -350000 & 275 & 261 & 208 \\
\hline 411 & UJ7307P & 004600 & -300000 & 216 & 205 & 168 \\
\hline & & & totals: & 1516 & 1387 & 1139 \\
\hline
\end{tabular}

$\mathrm{N1}=$ number in the high-probability subset

$\mathrm{N} 2$ = number after rejection of obvious contaminants

$\mathrm{N} 3$ = number with assigned redshift between 1.8 and 2.4

Our objects cover $\sim 28.3 \mathrm{deg}^{2}$ of each field. The limiting magnitudes are unknown because of the present lack of external data for calibration, but they should be $B \sim 20.5$ for the best plates; consequently, no significance should be attached to the variations in surface density from field to field.

Obvious contaminants were rejected; these are usually stars or surviving overlaps which, for special reasons, have slipped through the grading process.

A high-probability candidate must have an emission line - so a redshift can always be assigned. Redshifts were assigned on the assumption that the typically single lines are $\mathrm{Ly}-\alpha$, and the objects then restricted to the range 1.8 to 2.4 . The reason for the restriction is that 3-D PSA requires a comoving cuboid of points (see Webster 1982 and Clowes 1986), which can only be defined for a 
small range of redshifts if there is to be no serious distortion of scales. The range 1.8 to 2.4 contains a large fraction of the objects.

The way to define the comoving cuboid is given in Clowes (1986); its present-epoch volume is $\sim 390 \times 390 \times 1000 \mathrm{~h}^{-3} \mathrm{Mpc}^{3}$ for each of the fields, the largest dimension being in the redshift direction.

At this point it is useful to have an estimate of what fraction of the objects are definitely quasars. Visual prejudice applied to a film copy of each plate and 1-D plots of the objective-prism spectra suggests that the mean fraction corresponding to convincing quasars is $\sim 80 \%$. The true fraction could be higher still.

Thus, we expect that our final samples for 3-D PSA will contain a very low fraction of non-quasar contaminants, but we should also estimate in what fraction the assumption of $\mathrm{Ly}-\alpha$ is correct. Assumed Ly- $\alpha$ could really be CIV $\lambda 1549$, CIII] $\lambda 1909$, and MgII $\lambda 2798$. Hazard's (1985) histogram of redshifts is not ideal but gives the most relevant data: it suggests that the assumption will be correct in $30 / 43$ or $\sim 70 \%$ of cases. The true percentage could, perhaps, be as high as $80 \%$ simply because our candidates are of the highest grade.

The objective-prism redshifts are systematically too small by $\sim 0.04$ at $\mathrm{z} \sim 2.1$; however, this error is small and has been ignored. The random errors on the redshifts are such that the separation in the redshift direction of two objects carries an error of $\sim 30 \mathrm{Mpc}$ at $\mathrm{z} \sim 2.1$, which is much smaller than the mean separation. of objects.

The effect of non-quasar contaminants, misidentified lines, and redshift errors has been established from simulations.

\section{Results of the 3-D PSA}

Fig. 1 shows the results of combining the 3-D PSA statistics (the purely 1-D and 2-D terms are excluded) from the five fields. The figure plots $Q^{\prime}$ against $1 / \lambda$ for a bin size of $2.0 \mathrm{Gpc}^{-1}$, with error bars of $\pm \sigma$. Recall from Webster (1976) and Clowes (1986) that if the plot of $Q^{\prime}$ against $1 / \lambda$ suggests clustering on a scale of $\lambda_{c}$ then the PSA statistics are combined for $\lambda>\lambda_{c}\left(k<k_{c}\right)$, giving $Q$, to establish the significance of the clustering and to estimate the mean number of objects per cluster. For a random distribution the expectation values of $Q^{\prime}$ and $Q$ are 1 .

Simulations of our data show that, if we combine the PSA statistics from the five fields, we can detect clustering if only $\sim 6 \%$ of all quasars are paired on small scales. This value incorporates allowances for non-quasar contaminants, misidentified lines, and redshift errors.

There is no indication of clustering in Fig. 1.

\section{Comments}

The result of this clustering analysis is that there is convincing evidence for no 


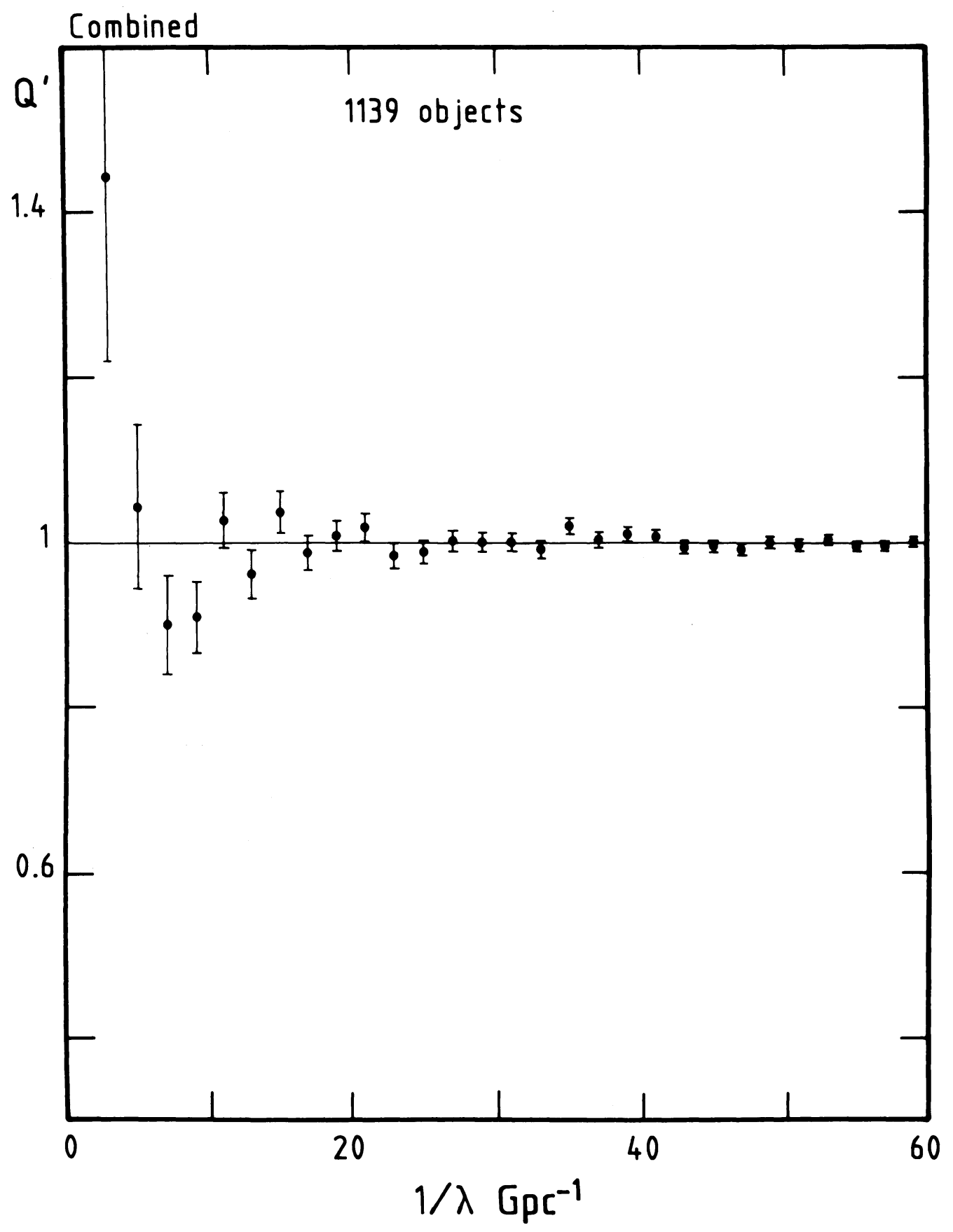

Figure 1. The 3-D PSA plot of $Q^{\prime}$ against $1 / \lambda$, with $1-D$ and $2-D$ terms excluded, for the combined statistics. The bin size is $2.0 \mathrm{Gpc}^{-1}$ and the error bars are $\pm \sigma$. 
clustering. If quasars are, in fact, physically clustered as pairs on the scale of superclusters (Oort 1983) then we can deduce that the fraction of our quasars participating in pairs is less than $\sim 6 \%$.

There have been other clustering analyses which cover the same small $\left(\sim 10 \mathrm{~h}^{-1}\right.$ $\mathrm{Mpc})$ to intermediate $\left(\sim 100 \mathrm{~h}^{-1} \mathrm{Mpc}\right)$ scales that are considered here, and they have divided into results of no clustering (eg Osmer 1981, Webster 1982, Clowes 1986) and results of possible clustering on small scales but not on intermediate scales (Shaver 1984, Boyle 1986).

Shaver's (1984) approach is to use the published positions and redshifts of quasars that are collected in a large, inevitably heterogeneous, compilation (Veron-Cetty \& Veron 1984). The heterogeneity makes comparison very difficult: the result could be real, and possibly attributable to low-redshift quasars only (Kruszewski 1986); it could, despite the allowances made, be caused by the selection effects of observation and publication that are inherent in a compilation.

Comparison with Boyle's (1986) result is rather easier. The quasars were discovered in an automated ultraviolet-excess survey, and the 3-D clustering analysis, using the autocorrelation method, of 169 quasars had the advantage of spectroscopic redshifts. The clustering result implies that $\sim 13 \%$ of quasars are paired on scales $<\sim 10 \mathrm{~h}^{-1} \mathrm{Mpc}\left(\mathrm{q}_{\mathrm{O}}=0.5\right)$, but it is also so noisy that a random distribution is not definitely excluded. A fraction of $\sim 13 \%$ would transform to $\sim 2-3 \%$ for our brighter quasars, and be below the detection threshold. Also, the mean redshift of Boyle's quasars is $\sim 1.5$ compared with $\sim 2.1$ for ours, which could be relevant.

Fewer than $\sim 6 \%$ of our quasars can be paired. To detect clustering still weaker than $\sim 6 \%$ the sensitivity of the analysis must be increased still further. In principle, this can be done quite easily by substituting spectroscopic redshifts for objective-prism redshifts. A quasar redshift survey would, in just one of our fields, improve the sensitivity to better than $3 \%$ for pairs of maximum separation $10 \mathrm{~h}^{-1} \mathrm{Mpc}$. There would also be the opportunity to investigate variations of clustering with epoch.

\section{Acknowledgments}

The staff of the Image and Data Processing Unit at ROE and of the UK Schmidt Telescope Unit at ROE and Coonabarabran are thanked for their help.

\section{$\underline{\text { References }}$}

Boyle, B.J., 1986. PhD thesis, University of Durham.

Clowes, R.G., Cooke, J.A. \& Beard, S.M., 1984. Mon. Not. R. astr. Soc., 207, 99.

Clowes, R.G., 1986. Mon. Not. R. astr. Soc., 218, 139.

Hazard, C., 1985. In "Active Galactic Nuclei", ed. Dyson, J.E., Manchester University Press.

Kruszewski, A., 1986. Preprint.

MacGillivray, H.T. \& Stobie, R.S., 1984. Vistas Astr., 27, 433. 
Oort, J.H., 1983. In "Quasars and Gravitational Lenses", 24th Liège Astrophysical Colloquium, Universite de Liège.

Osmer, P.S., 1981. Astrophys. J., 247, 762.

Shaver, P.A., 1984. Astron. Astrophys., 136, L9.

Véron-Cetty, M.-P. \& Véron, P., 1984. ESO scientific report no. 1.

Webster, A., 1976. Mon. Not. R. astr. Soc., 175, 61.

Webster, A., 1982. Mon. Not. R. astr. Soc., 199, 683.

\section{DISCUSSION}

BOYLE: You said you would only expect to find $6 \%$ of your QSOs in pairs at separations less than a few Mpc. Could you quantify the word 'few'?

CLOWES: About 5 . 